\title{
Differential introgression among loci across a hybrid zone of the intermediate horseshoe bat (Rhinolophus affinis)
}

\author{
Xiuguang Mao ${ }^{1,2}$, Guangjian Zhu', Libiao Zhang ${ }^{3}$, Shuyi Zhang ${ }^{1}$ and Stephen J Rossiter ${ }^{2 *}$
}

\begin{abstract}
Background: Hybrid zones formed by the secondary contact of divergent lineages represent natural laboratories for studying the genetic basis of speciation. Here we tested for patterns of differential introgression among three X-linked and 11 autosomal regions to identify candidate loci related to either reproductive isolation or adaptive introgression across a hybrid zone between two Chinese mainland subspecies of the intermediate horseshoe bat Rhinolophus affinis: R. a. himalayanus and R. a. macrurus.
\end{abstract}

Results: Our results support the previous suggestion that macrurus formed when a third subspecies ( $R$. a. hainanus) recolonized the mainland from Hainan Island, and that himalayanus is the ancestral taxon. However, this overall evolutionary history was not reflected in all loci examined, with considerable locus-wise heterogeneity seen in gene tree topologies, levels of polymorphism, genetic differentiation and rates of introgression. Coalescent simulations suggested levels of lineage mixing seen at some nuclear loci might result from incomplete lineage sorting. Isolation with migration models supported evidence of gene flow across the hybrid zone at one intronic marker of the hearing gene Prestin.

Conclusions: We suggest that phylogenetic discordance with respect to the species tree seen here is likely to arise via a combination of incomplete lineage sorting and a low incidence of introgression although we cannot rule out other explanations such as selection and recombination. Two X-linked loci and one autosomal locus were identified as candidate regions related to reproductive isolation across the hybrid zone. Our work highlights the importance of including multiple genomic regions in characterizing patterns of divergence and gene flow across a hybrid zone.

Keywords: Phylogenetic discordance, Adaptive introgression, Speciation, Rhinolophus

\section{Background}

Our understanding of the genetics of speciation has benefited greatly from studies of hybridizing species, both in the laboratory [1-3] and in the wild [4-6]. In the latter, hybrid zones - geographic regions where genetically distinct populations meet, mate and produce hybrids [7] - have been considered as 'natural laboratories for evolutionary studies' [8]. For evolutionary biologists, hybrid zones offer windows on evolutionary process [9], while they provide the divergent populations themselves with a means to interact with each other. Genes can exchange due to the semi-permeable nature of the genome [10], and this in

\footnotetext{
* Correspondence: s.j.rossiter@qmul.ac.uk

${ }^{2}$ School of Biological and Chemical Sciences, Queen Mary University of London, London E1 4NS, UK

Full list of author information is available at the end of the article
}

turn can result in variation in the level of introgression of alleles among different genomic regions [11-14].

Many processes can contribute to patterns of differential introgression, such as natural selection, genetic drift [15], varying recombination rates [16], linkage [4,17], sampling error, or a combination of these processes. Distinguishing these processes from each other is extremely difficult but may be possible with the aid of newly developed analytical approaches (e.g. genomic clines, [18]), and with sampling of increased numbers of loci across the genome that are being surveyed in multiple individuals.

Based on patterns of introgression, several different classes of genomic region in the hybridized genome have been identified. First, there are regions that resist introgression or show reduced levels of introgression, which are often considered to be, or be linked to, putative 'speciation genes' 
$[10,11,19]$. A number of such genes have been reported in model organisms; for example, in Drosophila $[3,20]$ and in the mouse (Mus) [2,21]. Second, there are regions that occur in the genomic backgrounds of both hybridizing species, which appear to be selectively neutral and able to flow freely across species boundaries. Finally, there are regions that introgress faster than neutral genes, and these are often considered to be advantageous or beneficial genes. Cases of such adaptive introgression may be promoted by positive selection [22], and have been documented in both plants $[23,24]$ and animals [25-27]. Recent genome scans conducted in mice have revealed that genes involved in olfaction and pheromone responses have undergone adaptive introgression across a hybrid zone, a result that was attributed to the importance of such genes in the survival and reproduction of these organisms [5].

By studying patterns of introgression in hybrid zones, candidate genomic regions that are either speciation genes, or linked to speciation genes, can be identified on the basis of reduced levels of introgression, whereas candidate genomic regions that are either beneficial genes, or linked to beneficial genes, can be detected from increased levels of introgression [4-6,11,15,28]. One advantage of this differential introgression approach is that it can help to identify candidate genomic regions that are likely to be related to reproductive isolation and/or adaptive introgression between hybridizing species even in the absence of any information about the phenotypes that such regions control. This is particularly useful for studies of wild populations from non-model organisms.

A hybrid zone between two subspecies of the intermediate horseshoe bat (Rhinolophus affinis) provides an opportunity to gain insights into the genomic regions likely to be responsible for reproductive isolation and/or adaptive introgression in these taxa. $R$. a. himalayanus and $R$. a. macrurus both occur on the Chinese mainland (see Figure 1a). Previous phylogeographic studies suggest that the mainland $R$. a himalayanus first colonized Hainan Island to form $R$. $a$. hainanus, which then underwent a post-glacial recolonization of the mainland to form $R$. a. macrurus [29,30]. The two mainland subspecies, himalayanus and macrurus, now form a hybrid zone in southeastern China. Earlier analyses of the mitochondrial control region, three nuclear genes and 14 microsatellites loci [30] suggested the occurrence of mitochondrial introgression but no nuclear introgression across the hybrid zone, although the absence of detected nuclear introgression may have reflected a lack of data.

To test for differential introgression across the hybrid zone in $R$. affinis, here we expand our sampling of the genome to include loci that might be subject to adaptive introgression. Unlike mice in which olfaction is the dominant sensory modality, horseshoe bats are auditory specialists that use a system of narrowband constant frequency echolocation in which the inner ear is finely tuned to the incoming echoes of the emitted call. These calls have evolved specifically for the detection of flying insects, with the call frequency influencing the prey size and habitat use. For this reason, putative echolocation (or hearing) genes [31] might be subject to adaptive introgression. To test this hypothesis in our study system, we analyzed polymorphism and genetic differentiation in regions from three echolocation genes (i.e. FoxP2, Kcnq4 and Prestin). The first of these is implicated in orofacial coordination and vocalisation - both of which are important in call production - and was shown to have undergone divergent selection in echolocating bats [32]. The latter two genes encode proteins involved in hair cell function, and both show extensive sequence convergence between echolocating bats and dolphins $[33,34]$. In comparison, we included three X-linked and five other autosomal regions that are not expected to have any roles in hearing or vocalisation. The inclusion of X-linked markers in our dataset allowed us to also test the hypothesis that the $\mathrm{X}$ chromosome contributes disproportionately to reproductive isolation compared to autosomes [35].

\section{Methods}

\section{Sampling and echolocation calls recording}

Individuals of all three subspecies ( $R$. a. himalayanus, $R$. a. macrurus and $R$. a. hainanus) sequenced in this study were sampled previously $[29,30]$ from across the range (Figure 1a). A wing membrane biopsy was taken from each individual following standard non-lethal sampling procedure that was approved by the National Animal Research Authority, East China Normal University (approval ID 20080209). One congeneric species $(R$. pearsoni) was included as an outgroup in the phylogenetic analyses. All DNA samples analyzed were isolated from 3-mm wing membrane biopsies using DNeasy kits (Qiagen).

Echolocation call frequency and body size are useful characters for distinguishing among closely related bat species [36]. To test for divergence in body size and call frequency among the three focal taxa, for a subset of bats we measured the forearm $(\mathrm{mm})$ using dial calipers, and recorded the echolocation call resting frequency $(\mathrm{kHz})$ using the Avisoft UltraSoundGate 116Hnb kit (Avisoft, Berlin, Germany). Prior to taxonomic comparisons, we also tested for sex differences among call frequencies, which have been reported in several horseshoe bat species. Spectrograms were analyzed using AvisoftSASLab Pro software (Avisoft) and the constant frequency of the second harmonic was extracted. 

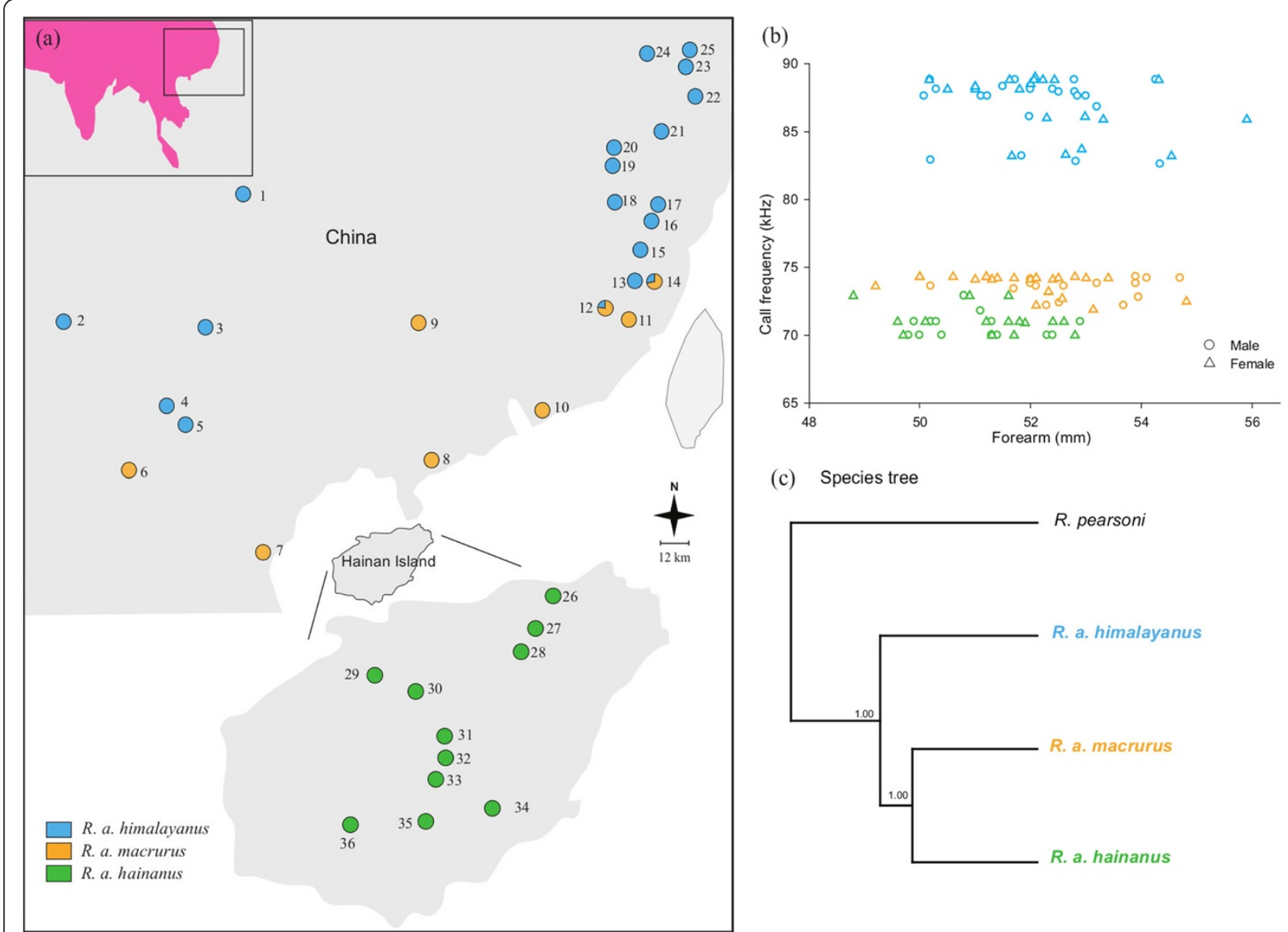

(c) Species tree

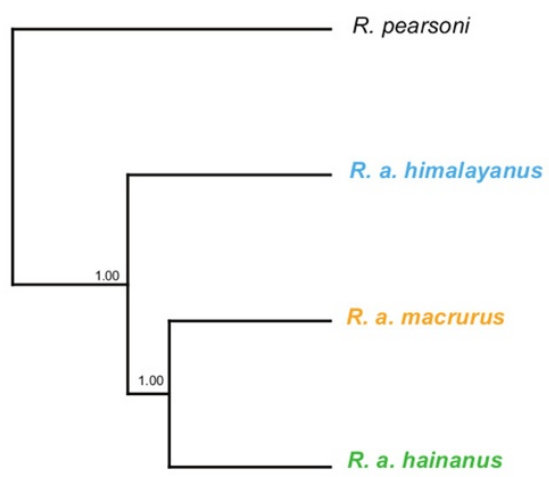

Figure 1 Sampling, morphological data and species tree. (a) Map showing the sampling localities of Rhinolophus affinis used in this study (modified from Mao et al. 2013 [30]). Populations are presented as circles in which individuals are coloured based on the subspecies membership (R. a. himalayanus: blue; R. a. macrurus: orange; R. a. hainanus: green); (b) Plot showing echolocation call frequency ( $\mathrm{kHz}$ ) and forearm (mm) data for a subset of bats from the three subspecies (R. a. himalayanus, $\mathrm{n}=43 ; R$. a. macrurus, $\mathrm{n}=39 ; R$. a. hainanus, $\mathrm{n}=32$ ). Downward and upward triangles correspond to male and female bats, respectively. Full details are provided in Additional file 3: Table S3; (c) Species tree based on all nuclear markers using BEST.

\section{Gene selection and sequencing}

Sequence data of Chd1, Sws 1 and Usp $9 x$ from individuals of himalayanus and macrurus were taken from our previous study [30]. To complement these data, here we also sequenced these genes in the hainanus island subspecies, and, for all three taxa, we obtained new sequence data from nine additional genes. First, we amplified the complete cytochrome $b(C y t b)$ gene using the primers and PCR conditions described in [37]. In addition, for each mtDNA clade (see Results), we also amplified introns of two X-chromosomal genes (Pola1 and Cx22) and six autosomal genes (Tg, Thy, H2a, Kcnq4, FoxP2 and Prestin). For FoxP2 and Prestin, we amplified the second and third introns of the former (hereafter called FoxP2-2 and -3) and four introns of the latter (hereafter called Prestin-4, $-8,-17$ and -18). Details of all markers examined here are provided in Table 1.
Polymerase chain reactions (PCRs) were performed in $50 \mu \mathrm{l}$ reaction mixtures containing 10-50 ng DNA, $0.25 \mathrm{mM}$ of each primer and $25 \mu \mathrm{l}$ Premix Taq polymerase (TaKaRa). The thermocycling profiles for $T g$, Thy and $U s p 9 x$ have been described previously [38-40]. For H2a, Pola1, Cx22, Prestin-4, $-8,-17$ and -18, FoxP2-2 and -3 , and $K c n q 4$, we used: $95^{\circ} \mathrm{C}$ for $5 \mathrm{~min}$; 34 cycles of $94^{\circ} \mathrm{C}$ for $30 \mathrm{~s}, 54-61^{\circ} \mathrm{C}$ for $40 \mathrm{~s}, 72^{\circ} \mathrm{C}$ for $90 \mathrm{~s} ; 72^{\circ} \mathrm{C}$ for $10 \mathrm{~min}$. Details of primer information and actual anneal temperatures for each marker have been provided in Additional file 1: Table S1. PCRs were carried out on a PTC-220 thermal cycler (Bio-Rad). DNA sequencing was undertaken with both primers on an ABI PRISM 3700 automated sequencer (Applied Biosystems). For nuclear sequences, when multiple heterozygous sites were present (i.e. more than one double peak was observed in the sequence chromatograms), haplotypes were resolved probabilistically using PHASE 2.1 [41] in the package DnaSP 
Table 1 Annotated function and chromosome information for molecular markers used in this study

\begin{tabular}{llll}
\hline ID & Description & Chromosome & Annotated function \\
\hline Cytb & Cytochrome $b$ & Mitochondrial & Oxidative phosphorylation \\
Chd1 & Chromodomain helicase DNA binding protein 1 & Chromatin binding \\
Sws1 & The short-wavelength-sensitive opsin gene & Autosome & Cognition \\
H2a & H2A histone family, member Y & Autosome & Chromatin binding \\
Thy & Thyrotropin & Autosome & Hormone activity \\
Tg & Thyroglobulin & Autosome & Hormone activity \\
Prestin & Solute carrier family 26, member 5 & Autosome & Hearing \\
FoxP2 & Forkhead box P2 & Autosome & Cognition \\
Kcnq4 & The voltage-gated potassium channel subfamily KQT member 4 & Autosome & Hearing \\
Usp9x & Ubiquitin specific protease 9 X & Autosome & Alternative splicing \\
Pola 1 & Polymerase (DNA directed) alpha 1 & X chromosome & Chromatin binding \\
CX22 & X-chromosomal open reading frame 22 & X chromosome & Alternative splicing \\
\hline
\end{tabular}

v5 [42]. Sequences were aligned using CLUSTAL_X 1.83 [43] and edited manually. All sequences generated in this study have been deposited in GenBank and accession numbers are given in Additional file 2: Table S2.

\section{Gene genealogies and species tree}

Phylogenetic relationships among the three subspecies were reconstructed based on the Cytb sequences using Bayesian inference (BI) implemented in MrBayes 3.1.2 [44]. To test for genealogical discordance across loci, we also performed a BI tree reconstruction for each nuclear region. For FoxP2, most individuals were sequenced at both FoxP2-2 and -3, and thus these two segments were concatenated as a single marker (hereafter called FoxP2). For Prestin, the DNA of some individuals was either too depleted or degraded for use in all segments of Prestin; thus each segment was analyzed separately. The best-fit substitution models for each region were determined in MODELTEST 3.0 [45] and are given in Table 2. We performed two simultaneous runs of Metropolis-coupled Markov chain Monte Carlo (MCMC) analysis with the substitution model parameters, each comprising four chains and 10 million generations. Trees and parameters were sampled every 100 generations, and the first $25 \%$ of the sampled trees were discarded as burn-in. Because gene genealogies at the population level are often difficult to represent by traditional phylogenetic trees [46], we also examined the relationship among haplotypes by constructing statistical parsimony networks in the package TCS version 1.21 [47].

Knowledge of the correct species tree is essential for understanding patterns of introgression. However, due to random lineage sorting, individual gene trees often differ from each other and from the species tree [48]. A Bayesian hierarchical model has been proposed to reconstruct the species tree by incorporating information from multiple gene trees $[49,50]$. We performed a Bayesian hierarchical model using the software BEST 2.0, which implements a MCMC algorithm to estimate the posterior distribution of species trees. For this analysis, all nuclear markers were included and model parameters for each marker were estimated using MODELTEST (Table 2). In total we included 14 individuals for which full sequence datasets were available: six himalayanus, four macrurus, three hainanus and one $R$. pearsoni as an outgroup. We performed two runs of Metropolis-coupled MCMC, each comprising four chains and 10 million generations. Trees and parameters were sampled every 100 generations, and the first $25 \%$ of the sampled trees were discarded as a burn-in.

Table 2 Substitution models determined by MODELTEST for each locus

\begin{tabular}{ll}
\hline Locus & Substitution models \\
\hline Cytb & $H K Y+I+G[I=0.7084, G=1.1615]$ \\
Cx22 & $H K Y$ \\
Usp9x & $H K Y$ \\
Pola1 & $H K Y+I+G[I=0.8750, G=0.6921]$ \\
Chd1 & $H K Y$ \\
H2a & $\mathrm{K} 81 \mathrm{uf}+\mathrm{I}+\mathrm{G}[\mathrm{I}=0.7127, \mathrm{G}=0.7697]$ \\
Sws1 & $\mathrm{HKY}+\mathrm{G}[\mathrm{G}=0.0061]$ \\
Thy & $\mathrm{K} 81 \mathrm{uf}+\mathrm{I}+\mathrm{G}[\mathrm{I}=0.8111, \mathrm{G}=0.8005]$ \\
Tg & $\mathrm{K} 80+\mathrm{G}[\mathrm{G}=0.0012]$ \\
Prestin-4 & $\mathrm{HKY}+\mathrm{I}+\mathrm{G}[\mathrm{I}=0.8206, \mathrm{G}=0.6954]$ \\
Prestin-8 & $\mathrm{HKY}+\mathrm{I}+\mathrm{G}[\mathrm{I}=0.8333, \mathrm{G}=0.8597]$ \\
Prestin-17 & $\mathrm{HKY}+\mathrm{I}+\mathrm{G}[\mathrm{l}=0.8838, \mathrm{G}=0.7198]$ \\
Prestin-18 & $\mathrm{HKY}+\mathrm{G}[\mathrm{G}=0.0099]$ \\
FoxP2 & $\mathrm{K} 81 \mathrm{uf}+\mathrm{I}+\mathrm{G}[\mathrm{l}=0.7737, \mathrm{G}=0.8948]$ \\
Ken94 & $\mathrm{HKY}$ \\
\hline
\end{tabular}




\section{Analyses of polymorphism, genetic differentiation and neutrality}

For each nuclear locus and each subspecies, we calculated the nucleotide diversity $(\pi)$ using DnaSP, which was also used to count the number of polymorphic sites, fixed and shared mutations between pairs of subspecies. To assess whether genetic differentiation was lower among the two mainland subspecies, as would be expected from introgression in the hybrid zone, we calculated $\mathrm{F}_{\mathrm{ST}}$ [51] at each locus between each pair of taxa in the software ARLEQUIN 3.5 [52]. Significance was assessed based on 1000 permutations.

Deviations from the neutral model for each locus were assessed by estimating Tajima's D [53] in ARLEQUIN with significance assessed by 1000 permutations. Multilocus neutrality tests were conducted based on the HudsonKreitman-Aguade (HKA) method [54] in DnaSP.

\section{Coalescent simulations of incomplete lineage sorting}

Phylogenetic reconstructions of several nuclear loci revealed a paraphyletic relationship between himalayanus and [macrurus + hainanus] (see Results). Because such mixing could theoretically arise from either introgression or incomplete lineage sorting, we undertook coalescent simulations to test for a possible contribution of incomplete sorting to the patterns observed. We followed the method outlined in [55], with modifications described in [56]. For nuclear genes (i.e. Pola1, Sws1, Thy, $\mathrm{Tg}, \mathrm{H} 2 a, \mathrm{Kcnq} 4$, Prestin-4, -8 and -18) that showed mixing between the himalayanus and [macrurus + hainanus] clades, we used Mesquite v2.5 [57] to calculate Slatkin \& Maddison's $s$ statistic [58], a measure of the degree of lineage sorting assuming no gene flow after splitting. Given two defined lineages, a $s$ value of 1 is expected for a locus showing complete reciprocal monophyly, while a value of $>1$ indicates mixing. Thus for each locus showing an observed $s$ value of $>1$, we used Mesquite to simulate 1,000 coalescent trees under the incomplete lineage sorting model with no migration within the population tree. Then, sequence data were simulated from the coalescent trees using a model of substitution estimated from the empirical data using MODELTEST excluding the outgroup (see Additional file 3: Table S3). The scale factor for each locus was determined by testing several values until mean sequence divergence values within lineages were similar between simulated and empirical data (see Additional file 3: Table S3). Finally, PAUP* [59] was used to reconstruct majority-rule consensus trees from sequence matrices using heuristic parsimony searches. For each locus, we generated a null distribution of $s$ values, against which the empirical value was compared and considered as significant if it fell outside of the $95 \%$ confidence intervals.

For all coalescent simulations, the divergence time between himalayanus and [macrurus + hainanus] was determined as 550000 years ago (i.e. 275000 generations based on a generation time of two years for horseshoe bats) using a sequence divergence of 0.011 (introgressed haplotypes were excluded in this analysis) and the divergence rate of $0.02 / \mathrm{Mya}$ for cytochrome $\mathrm{b}$; effective population size $\left(\mathrm{N}_{\mathrm{e}}\right)$ was estimated from $\Theta$ values using MIGRATE-N version 3.3.2 [60]. Each search contained 20 short chains with 5000 sampled genealogies and 2 long chains with 50,000 sampled genealogies. Genealogies were 200 steps apart and the first 10,000 were discarded as burn-in. Two runs were performed to ensure convergence of parameter estimates. The $\Theta$-values were converted to $\mathrm{N}_{e}$ using the formula $\Theta=4 \mathrm{~N}_{\mathrm{e}} \mathrm{u}$ ( $\mathrm{u}$ is mutation rate). The mutation rates of each nuclear locus were determined by calculating the ratio of the average distance between himalayanus and [macrurus + hainanus] for each locus and that for cytb $\left(1 \times 10^{-8}\right.$ per site per year $)$. Simulations were conducted using an $\mathrm{N}_{\mathrm{e}}$ based on the maximum likelihood estimates of $\Theta$, as well as the $95 \%$ upper and lower percentiles.

\section{Estimates of gene flow}

To further test for introgression, we estimated levels of gene flow between himalayanus and macrurus by implementing isolation-with-migration (IM) models [61] in the program IMa2 $[62,63]$. We repeated the IM analysis for each of the nine loci (Pola1, Sws1, Thy, Tg, H2a, Kcnq4, Prestin-4, -8 and -18) showing evidence of mixing between himalayanus and its daughter taxa. The IM model assumes that each locus is free from recombination and selectively neutral. We tested for recombination using the four-gamete test [64] in DnaSP and only the segments without recombination were used. Neutrality tests have been conducted above. Several preliminary runs were performed to establish upper bounds on prior distributions. A final run was conducted with 200000 genealogies at every 100 steps after a burn-in of $10^{6}$ steps including twenty Metropolis-coupled chains with a geometric heating scheme: -hfg -hn20 -ha0.96 -hb0.9. A total of 200000 genealogies were used to perform likelihood ratio tests of the nested models for migration rates [62].

\section{Results}

\section{Echolocation call frequency and species tree}

Rhinolophus affinis showed no sex differences in echolocation call frequency, however, significant divergence was observed among the taxa (see Figure $1 \mathrm{~b}$ and Additional file 4: Table S4). Specifically, the call frequencies of himalayanus $(87.12 \pm \mathrm{SD} 2.04 \mathrm{kHz})$ were significantly higher than those of both macrurus (macrurus: $73.68 \pm$ $0.74 \mathrm{kHz} ; \mathrm{t}=39.020, \mathrm{df}=83, \mathrm{P}<0.001$ ) and hainanus $(70.85 \pm 0.94 \mathrm{kHz} ; \mathrm{t}=42.102, \mathrm{df}=76, \mathrm{P}<0.001)$. Call frequencies differences between macrurus and hainanus 
were slight but also significant $(t=14.189, \mathrm{df}=69, \mathrm{P}<$ $0.001)$. The sister relationship between macrurus and hainanus, and the ancestral status of himalayanus, were further supported by the species tree estimated by BEST based on all nuclear regions (Figure 1c).

\section{MtDNA tree and network}

The final alignment (1023 bp) of 73 Cytb sequences identified 35 haplotypes among the three taxa. No premature stop codons were observed at this gene, suggesting that it is functional. A Bayesian tree and network based on all Cytb haplotypes gave a similar pattern to an earlier tree based on the Control Region (CR) [29]. Specifically, haplotypes of macrurus were nested within a predominantly hainanus clade (Figure 2) supporting the previous suggestion that macrurus originated from the recolonisation of hainanus onto the mainland. In this tree, most himalayanus haplotypes appeared to be ancestral with respect to these other taxa, and formed two clusters with one corresponding to a specific geographical region (see [30]). However, several individuals' Cytb haplotypes - hap1, hap10 and hap11 - were classified with the [macrurus + hainanus] clade, supporting mtDNA introgression between the two mainland subspecies (see also $[29,30])$.

\section{Discordance in genealogical topologies among nuclear loci} To test for phylogenetic discordance among genomic regions, phylogenetic trees and networks were reconstructed for all nuclear regions examined (Figure 3). Five regions (i.e. Cx22, Usp9x, Chd1, Prestin-17, and Foxp2) recovered phylogenetic relationships among the three taxa that were similar to those seen in the mtDNA and species trees, with macrurus and hainanus haplotypes mixed and highly divergent from himalayanus haplotypes. However, eight regions (i.e. Pola1, Sws1, Thy, Tg, H2a, Prestin-4, Prestin-8, and Prestin-18) produced genealogies in which some haplotypes of himalayanus were classified with [macrurus + hainanus]. In particular, in Pola1 and Sws1, all himalayanus haplotypes clustered with some of the macrurus haplotypes with high posterior probabilities (Figure 3), indicating possible gene flow between these two mainland subspecies. One gene (Kcnq4) showed mixed haplotypes among all three subspecies (Figure 3), possibly due to its low mutation rate.

Polymorphisms and genetic differentiation, and neutral test The proposed recent origin of macrurus by the recolonization of hainanus onto the mainland would be expected to lead to lower levels of nucleotide diversity in macrurus than in either hainanus or himalayanus. However, locus-wise nucleotide diversity was not seen to differ significantly between macrurus and hainanus $(\mathrm{t}=-1.4055, \mathrm{df}=13, \mathrm{P}=0.1833$, paired $\mathrm{t}$-test $)$ with $50 \%$ of markers (Pola1, H2a, Tg, Prestin-4, Prestin-18, FoxP2 and Kcnq4; see Table 3) showing higher pairwise nucleotide diversity values in the former taxon.

Under a scenario of lineage sorting with no gene flow during the divergence of the three taxa, the most recently diverged hainanus and macrurus should share more ancestral polymorphisms. Consistent with this expectation, no loci showed fixed differences between hainanus and macrurus compared with nine loci between himalayanus and macrurus, and ten loci between himalayanus and hainanus (Table 4). Likewise, levels of genetic differentiation were significantly lower between hainanus and macrurus (average $\mathrm{F}_{\mathrm{ST}}=0.23$ ) than between himalayanus and macrurus (average $\mathrm{F}_{\mathrm{ST}}=0.62, \mathrm{t}=-5.063$, $\mathrm{df}=28, \mathrm{P}<0.0001)$, and between himalayanus and hainanus (average $\mathrm{F}_{\mathrm{ST}}=0.66, \mathrm{t}=-5.561, \mathrm{df}=28, \mathrm{P}<$ 0.0001). On the other hand, six loci - Pola1, Sws1, Thy, Foxp2, Prestin-4, and Prestin-8 - showed lower differentiation between himalayanus and macrurus than between himalayanus and hainanus $(\mathrm{t}=-3.531, \mathrm{df}=10, \mathrm{P}<0.05)$, again suggesting possible introgression between himalayanus and macrurus. The multilocus HKA test suggested no departure from neutrality for any loci, although the standard neutrality test based on Tajima's D was significant for Chd1 in himalayanus (Table 3).

\section{Coalescent simulations of incomplete lineage sorting}

Four (Sws1, Pola1, H2a and Prestin-8) of the nine loci that showed mixing between the himalayanus and [macrurus + hainanus] clades were characterized by $s$-statistic values of 1 , indicating complete reciprocal monophyly at these loci. The remaining five loci were characterized by $s$-statistic values of $>1$ (the $s$ value for Prestin-4, Thy, Tg and Kcnq4 was 2 and for Prestin-18 was 3), indicating a lack of reciprocal monophyly between himalayanus and [macrurus + hainanus]. The scenario of incomplete lineage sorting with no migration could not be rejected in four of loci (Prestin-4, Thy, Kcnq4 and Prestin-18) with the values of $s$ falling within the $95 \%$ confidence intervals generated from simulation tests (Figure 4). In contrast, the $s$-value for $T g$ was significantly small, falling in the lower $5 \%$ tail of values, suggesting that it probably did not reflect incomplete lineage sorting.

\section{Estimates of gene flow}

We compared the fit of nested models with zero gene flow for each of the nine loci using IMa2. The likelihood ratio test rejected the model with zero gene flow only at Prestin-4 from macrurus to himalayanus $(\mathrm{P}=0.043)$ (see details in Additional file 5: Table S5).

\section{Discussion}

In this study the species tree estimated using 14 loci under a Bayesian hierarchical model and the phylogeny 


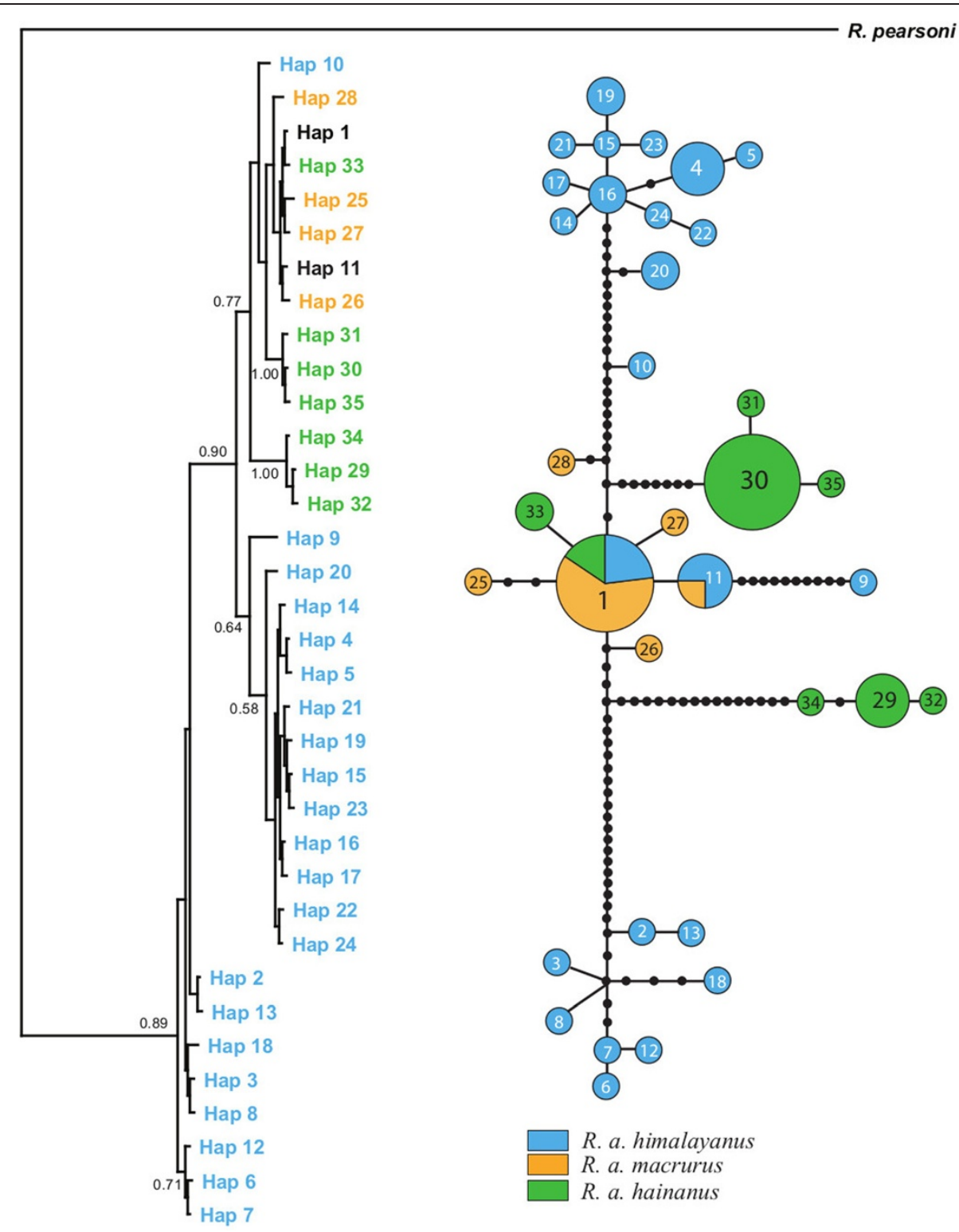

Figure 2 Bayesian inference (BI) tree and statistical parsimony network based on sequences from Cytochrome b (Cytb). In trees, node support is indicated with Bayesian posterior probabilities and only posterior probabilities over 0.5 are shown. Shared haplotypes between pairs of subspecies are shown in black. In networks, each circle represents a single haplotype and the area of circle size is scaled by haplotype frequency.

The filled black circles represent missing or unsampled haplotypes.

constructed from mitochondrial DNA, together with call frequency variation, supports our earlier proposal that himalayanus colonized Hainan Island to form hainanus, which later recolonized the mainland forming macrurus $[29,30]$. The recent origin of macrurus from hainanus was also supported by its comparatively lower average genetic differentiation (Table 3) and almost no fixed differences seen in nuclear markers here (Table 4).

Despite these patterns, the same evolutionary history was not suggested by all 14 nuclear loci. Indeed, nine loci showed evidence of mixing between himalayanus and its daughter taxa based on their genealogical topologies.
However, four of those loci (Sws1, H2a, Pola1 and Prestin8 ) gave the $s$-statistic value of 1 , indicating complete reciprocal monophyly in these loci. This was confirmed by the IMa2 analysis that indicated that the model of zero gene flow could not be rejected in these loci. In the remaining five loci (Prestin-4, Thy, Tg, Kcnq4 and Prestin-18) with the $s$-statistic values $>1$, the absence of reciprocal monophyly might have plausibly arisen from incomplete lineage sorting although low levels of introgression might also contribute to this pattern, e.g. at Prestin-4. Additionally, differences in locus-specific recombination rates can also lead to variation in levels of genetic differentiation and gene flow $[16,65,66]$. 


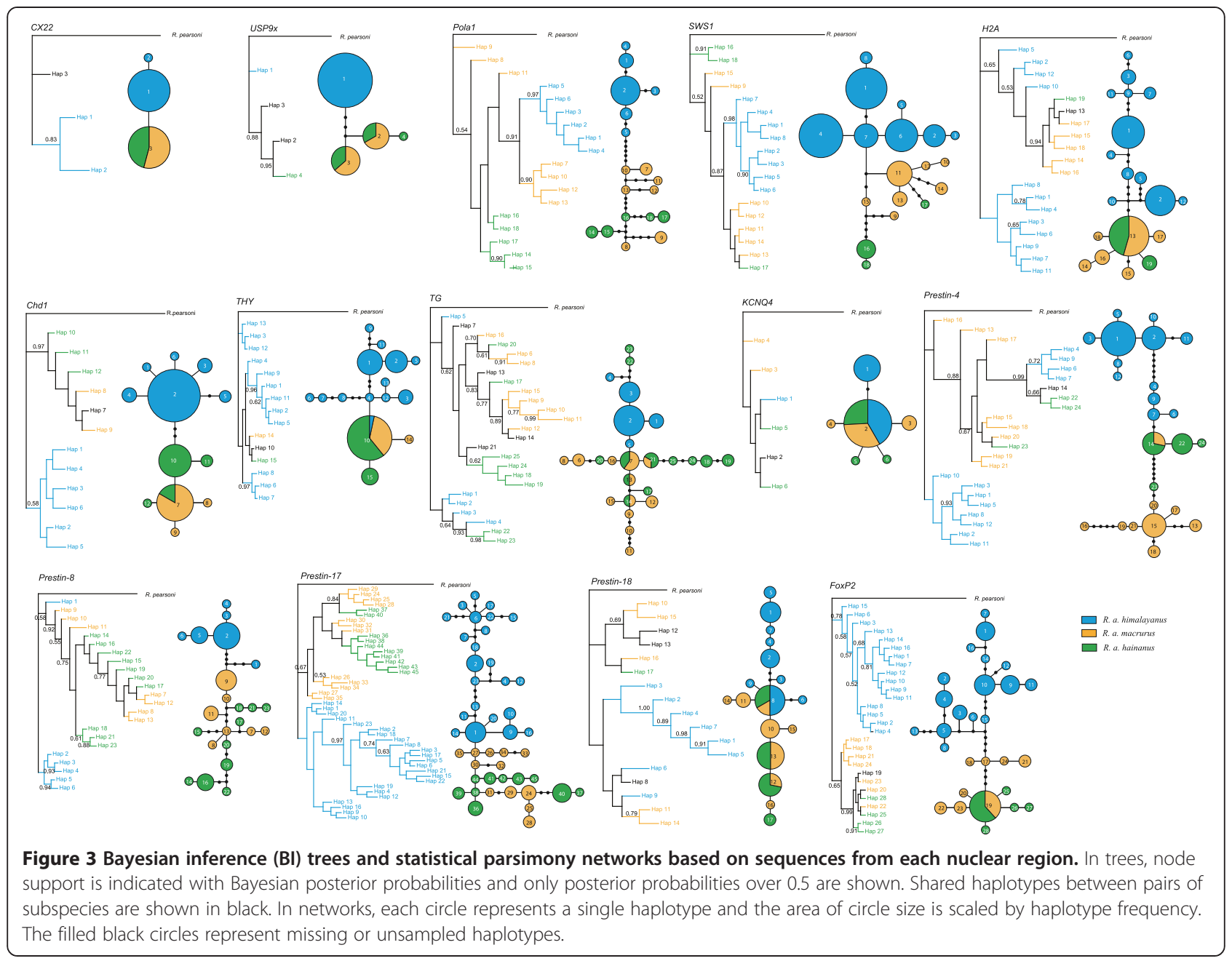

Our findings add to several recent studies of closely related taxa that have also shown that genealogical patterns and/or levels of genetic differentiation and gene flow can vary considerably across different genomic regions [67-69].

Of the three X-linked markers studied, two (Cx22 and Usp $9 x)$ showed extremely low levels of nucleotide diversity $(\pi<0.12 \%)$ within all three subspecies, alongside high genetic differentiation $\left(\mathrm{F}_{\mathrm{ST}}>0.94\right)$ between himalayanus and the other taxa, in accordance with a reciprocal monophyly for himalayanus and [macrurus + hainanus]. Such signatures could arise from selective sweeps operating to reduce genetic diversity within each taxon while fixing divergent alleles in different taxa [70]. These patterns are consistent with the idea that the X-chromosome plays a greater role than autosomes in reproductive isolation [4,5,71]. In contrast, however, the third X-linked locus (Pola1) showed highest nucleotide diversity in macrurus (the youngest taxon) than any of the other markers. Moreover, this locus also showed both a closer phylogenetic relationship and low estimated genetic differentiation between himalayanus and macrurus than between macrurus and hainanus, both of which likely resulted from either incomplete lineage sorting or introgression between himalayanus and macrurus. However, both of these two scenarios were not supported by the estimated $s$ value of 1 , or by the nested migration models performed in IMa2. Thus, other processes such as recombination might also contribute to the patterns observed in Pola1 (see also [68]).

The IMa2 analysis indicated that the model with zero gene flow could be rejected for Prestin-4, supporting our original hypothesis that candidate echolocation genes may be subject to adaptive introgression (see Background). On the other hand, the other two candidate echolocation genes (FoxP2 and Kcnq4) examined showed no evidence of introgression across the hybrid zone and, therefore, we cannot confidently associate the introgression of Prestin with its role in echolocation. Additional candidate hearing and/or echolocation genes must be studied for a more thorough assessment of whether there are differences in levels of introgression between 
Table 3 Estimates of polymorphism (nucleotide diversity $(\pi)$ and Tajima's D) within each subspecies and genetic differentiation $\left(F_{S T}\right)$ between pairs of subspecies for each nuclear region

\begin{tabular}{|c|c|c|c|c|c|c|c|c|c|c|c|c|}
\hline \multirow[b]{2}{*}{ Locus } & \multicolumn{3}{|l|}{$N$} & \multicolumn{3}{|l|}{$\pi$} & \multicolumn{3}{|c|}{ Tajima's D } & \multicolumn{3}{|l|}{$\mathrm{F}_{\mathrm{ST}}$} \\
\hline & him & mac & hai & him & mac & hai & him & $m a c$ & hai & him-mac & hai-mac & him-hai \\
\hline$C \times 22$ & 28 & 13 & 12 & 0.017 & 0 & 0 & -1.156 & 0 & 0 & 0.95 & 0 & 0.95 \\
\hline Usp9x & 42 & 12 & 9 & 0 & 0.079 & 0.116 & 0 & 1.381 & 0.196 & 0.94 & -0.06 & 0.94 \\
\hline Polar & 20 & 11 & 8 & 0.287 & 1.187 & 0.404 & -0.509 & 0.823 & 1.293 & 0.67 & 0.34 & 0.81 \\
\hline Chd1 & 42 & 15 & 17 & 0.067 & 0.036 & 0.089 & $-1.726^{*}$ & -1.503 & 0.103 & 0.91 & 0.61 & 0.86 \\
\hline $\mathrm{H} 2 \mathrm{a}$ & 25 & 15 & 10 & 1.019 & 0.289 & 0.097 & 1.064 & -0.931 & 0.426 & 0.54 & 0.09 & 0.54 \\
\hline Sws 1 & 48 & 12 & 7 & 0.353 & 0.267 & 0.416 & 0.944 & -1.288 & $-1.576^{*}$ & 0.54 & 0.62 & 0.77 \\
\hline Thy & 17 & 11 & 17 & 0.759 & 0.041 & 0.073 & 0.658 & -1.129 & 0.292 & 0.47 & 0.08 & 0.53 \\
\hline $\mathrm{Tg}$ & 20 & 10 & 10 & 0.213 & 0.895 & 0.802 & -1.156 & 0.170 & -0.108 & 0.56 & 0.14 & 0.50 \\
\hline Prestin-4 & 23 & 12 & 9 & 0.518 & 0.579 & 0.284 & -0.133 & -1.062 & -1.446 & 0.62 & 0.64 & 0.73 \\
\hline Prestin-8 & 14 & 9 & 8 & 0.193 & 0.544 & 0.547 & -1.246 & 1.179 & 0.637 & 0.59 & 0.23 & 0.71 \\
\hline Prestin-17 & 14 & 9 & 10 & 0.646 & 0.439 & 0.567 & 0.136 & 0.087 & 1.876 & 0.36 & 0.26 & 0.38 \\
\hline Prestin-18 & 13 & 11 & 9 & 0.784 & 0.435 & 0.330 & 1.103 & 0.185 & 1.456 & 0.43 & 0.06 & 0.46 \\
\hline FoxP2 & 23 & 12 & 9 & 0.315 & 0.204 & 0.054 & 0.486 & 0.327 & -1.385 & 0.65 & 0.17 & 0.73 \\
\hline Kcnq4 & 16 & 11 & 8 & 0.164 & 0.081 & 0.064 & 1.999 & -0.532 & -1.401 & 0.31 & 0.06 & 0.28 \\
\hline
\end{tabular}

$\mathrm{N}$ is the number of individuals analyzed for each nuclear region within each subspecies. him represents himalayanus; mac represents macrurus; hai represents hainanus. ${ }^{*} \mathrm{P}<0.05$.

hearing genes and other genes in these bats. Indeed, tests for selection for this region of Prestin were not significant, suggesting that the possible occurrence of introgression in Prestin-4 may in fact be due to neutral gene flow although we cannot reject the alternative possibility of linkage to beneficial genes [27,72,73]. It was noteworthy that the scenario of incomplete lineage sorting could not be ruled out completely, and it is possible that the parapyhly pattern at this locus might have been shaped by a combination of both introgression and incomplete lineage sorting. Finally, we were unable to assess the effects of recombination rate on patterns of introgression $[16,68]$ due to lack of detailed knowledge of the genomic locations of the genes examined, such as whether they occur within rearranged or colinear regions of the genome. Beside the possible occurrence of gene flow across the hybrid zone, data from levels of polymorphism, genetic differentiation and genealogical

Table 4 Polymorphism and fixed difference between pairs of subspecies

\begin{tabular}{|c|c|c|c|c|c|c|c|c|c|}
\hline \multirow[b]{2}{*}{ Locus } & \multicolumn{3}{|c|}{ Polymorphic sites } & \multicolumn{3}{|c|}{ Fixed differences } & \multicolumn{3}{|c|}{ Shared mutations } \\
\hline & him-mac & hai-mac & him-hai & him-mac & hai-mac & him-hai & him-mac & hai-mac & him-hai \\
\hline$C \times 22$ & 2 & 0 & 2 & 1 & 0 & 0 & 0 & 0 & 0 \\
\hline Usp9x & 2 & 2 & 3 & 1 & 0 & 1 & 0 & 1 & 0 \\
\hline Polar & 17 & 16 & 12 & 1 & 0 & 4 & 3 & 2 & 1 \\
\hline Chd 1 & 12 & 4 & 11 & 4 & 0 & 3 & 0 & 0 & 0 \\
\hline $\mathrm{H} 2 \mathrm{a}$ & 20 & 7 & 16 & 1 & 0 & 1 & 1 & 0 & 0 \\
\hline Sws 1 & 16 & 13 & 17 & 1 & 0 & 2 & 0 & 3 & 0 \\
\hline Thy & 11 & 2 & 12 & 0 & 0 & 0 & 1 & 0 & 0 \\
\hline $\operatorname{Tg}$ & 19 & 21 & 17 & 0 & 0 & 1 & 0 & 5 & 3 \\
\hline Prestin-4 & 22 & 17 & 18 & 0 & 0 & 2 & 6 & 7 & 3 \\
\hline Prestin-8 & 16 & 14 & 17 & 1 & 0 & 2 & 1 & 5 & 2 \\
\hline Prestin-17 & 27 & 14 & 25 & 0 & 0 & 0 & 4 & 7 & 4 \\
\hline Prestin-18 & 14 & 6 & 11 & 0 & 0 & 0 & 1 & 3 & 1 \\
\hline FOXP2 & 30 & 13 & 29 & 4 & 0 & 8 & 0 & 0 & 0 \\
\hline Kcnq4 & 4 & 4 & 4 & 0 & 0 & 0 & 0 & 0 & 0 \\
\hline
\end{tabular}

him represents himalayanus; mac represents macrurus; hai represents hainanus. 


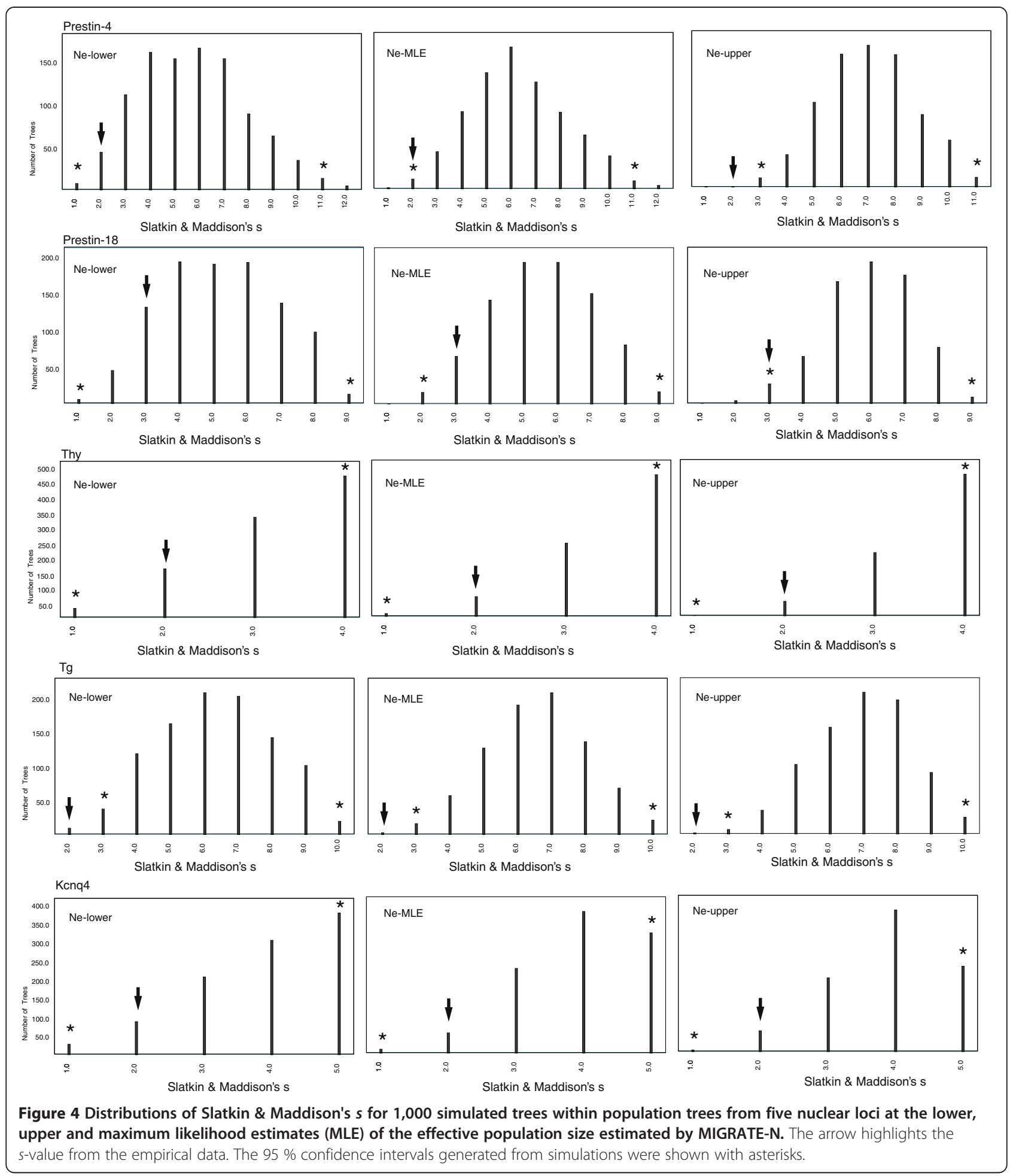

topology suggested that locus Chd 1 may represent a candidate region associated with reproductive isolation, either directly or via linkage to another locus.

Detected possible gene flow between himalayanus and macrurus at Prestin conflicts with our earlier findings for which no nuclear gene flow was inferred on the basis of reciprocal monophyletic tree topologies despite extensive mtDNA introgression [29,30]. Interestingly three nuclear genes (Chd1, Sws1 and Usp $9 x$ ) did not show evidence of gene flow in this study. Given 
these differences among loci, our results highlight the importance of including multiple genomic regions in order to characterize patterns of introgression across a hybrid zone. This differential introgression pattern across loci observed here has been widely documented in other taxa including both plants (e.g. sunflowers [24] and trees [74]) and animals (e.g. tiger salamanders [25], mice [26], butterflies [27], and crickets [75]. It is pertinent that a recent study has shown that this heterogeneity among loci can affect the inference of the history of speciation if it is not taken into account [76].

\section{Conclusions}

By comparing patterns of divergence and gene flow among loci we identified several regions with putative roles in either reproductive isolation (e.g. Cx22, Usp $9 x$ and Chd1) or adaptive introgression (e.g. Prestin) in the hybrid zone between himalayanus and macrurus. However, incomplete lineage sorting, as an alternative scenario for introgression, could not be ruled out completely in this study. Nonetheless a fuller understanding of the factors driving the process of differential introgression will benefit from the number of markers used and knowledge of genomic locations or functional/linkage relationships among those markers. Regardless, our findings add to mounting evidence that caution must be exercised when drawing conclusions about the occurrence of nuclear introgression on the basis of a small number of loci.

\section{Additional files}

Additional file 1: Table S1. Detailed information on the sequences of the primers, anneal temperature in PCR and references for each marker.

Additional file 2: Table S2. List of the GenBank accession numbers for all the sequences included in this study.

Additional file 3: Table S3. Detailed information of the forearm and echolocation call frequency for each individual used in this study.

Additional file 4: Table S4. Parameters used for nuclear markers in coalescent simulations.

Additional file 5: Table S5. Tests of nested models for migration rates between himalayanus and macrurus based on the full data set.

\section{Competing interests}

The authors declare that they have no competing interests.

\section{Authors' contributions}

XM, SZ, SR conceived the project. XM conducted the experiments and the data analysis and wrote the draft. GZ and LZ collected samples. SR edited the draft. SZ provided funding support. All authors read and approved the final manuscript.

\section{Acknowledgements}

We thank Jeffrey Feder and three anonymous reviewers whose comments improved the manuscript. This work was funded by a grant awarded to $\mathrm{S}$. Zhang under the Key Construction Program of the National "985" Project and "211" Project. X. Mao was supported by a Marie Curie International Incoming Fellowship and SJR by a Royal Society Research Fellowship (UK).

\section{Author details}

${ }^{1}$ Institute of Molecular Ecology and Evolution, East China Normal University, Shanghai 200062, China. ${ }^{2}$ School of Biological and Chemical Sciences, Queen Mary University of London, London E1 4NS, UK. ${ }^{3}$ Guangdong Entomological Institute, 105 Xingang Xilu, Haizhu Guangzhou, Guangdong Province 510260, China.

Received: 28 March 2014 Accepted: 4 July 2014

Published: 9 July 2014

\section{References}

1. Ting CT, Tsaur SC, Wu ML, Wu Cl: A rapidly evolving homeobox at the site of a hybrid sterility gene. Science 1998, 282(5393):1501-1504.

2. Mihola O, Trachtulec Z, Vlcek C, Schimenti JC, Forejt J: A mouse speciation gene encodes a meiotic histone H3 methyltransferase. Science 2009, 323(5912):373-375.

3. Phadnis N, Orr AH: A single gene causes both male sterility and segregation distortion in Drosophila hybrids. Science 2009, 323(5912):376-379.

4. Payseur BA, Krenz JG, Nachman MW: Differential patterns of introgression across the $\mathrm{X}$ chromosome in a hybrid zone between two species of house mice. Evolution 2004, 58(9):2064-2078.

5. Teeter KC, Payseur BA, Harris LW, Bakewell MA, Thibodeau LM, O'Brien JE, Krenz JG, Sans-Fuentes MA, Nachman MW, Tucker PK: Genome-wide patterns of gene flow across a house mouse hybrid zone. Genome Res 2008, 18:67-76.

6. Teeter KC, Thibodeau LM, Gompert Z, Buerkle CA, Nachman MW, Tucker PK: The variable genomic architecture of isolation between hybridizing species of house mice. Evolution 2010, 18(2):462-475.

7. Barton NH, Hewitt GM: Analysis of hybrid zones. Annu Rev Ecol Syst 1985, 16:113-148.

8. Hewitt GM: Hybrid zones-natural laboratories for evolutionary studies. Trends Ecol Evol 1988, 3(7):158-167.

9. Harrison RG: Hybrid zones: windows on evolutionary processes. In Oxford surveys in evolutionary biology vol 7. Edited by Futuyma D, Antonovics J. Oxford: Oxford University Press; 1990

10. Wu C-I: The genic view of the process of speciation. J Evol Biol 2001, 14(6):851-865

11. Dopman EB, Pérez L, Bogdanowicz SM, Harrison RG: Consequences of reproductive barriers for genealogical discordance in the European corn borer. Proc Natl Acad Sci USA 2005, 102(41):14706-14711.

12. Kronforst MR, Young LG, Blume LM, Gilbert LE: Multilocus analyses of admixture and introgression among hybridizing Heliconius butterflies. Evolution 2006, 60(6):1254-1268.

13. Maroja LS, Andres JA, Harrison RG: Genealogical discordance and patterns of introgression and selection across a cricket hybrid zone. Evolution 2009, 63(11):2999-3015.

14. Ohshima I, Yoshizawa K: Differential introgression causes genealogical discordance in host races of Acrocercops transecta (Insecta: Lepidoptera). Mol Ecol 2010, 19(10):2106-2119.

15. Payseur BA: Using differential introgression in hybrid zones to identify genomic region involved in speciation. Mol Ecol Resour 2010, 10(5):806-820.

16. Nachman MW, Payseur BA: Recombination rate variation and speciation: theoretical predictions ans emperical results from rabbits and mice. Proc R Soc London B Biol Sci 2012, 367(1587):409-421.

17. Rieseberg LH, Whitton HJ, Gardner K: Hybriz ones and the genetic architecture of a barrier to gene flow between two sunflower species. Genetics 1999, 152(2):713-727.

18. Gompert Z, Buerkle CA: Bayesian estimation of genomic clines. Mol Ecol 2011, 20(10):2111-2127.

19. Noor M, Feder JL: Speciation genetics: evolving approaches. Nat Rev Genet 2006, 7:851-861.

20. Brideau NJ, Flores HA, Wang J, Maheshwari S, Wang X, Barbash DA: Two Dobzhansky-Muller genes interact to cause hybrid lethality in Drosophila. Science 2006, 314(5803):1292-1295.

21. Payseur BA, Nachman M: The genomics of speciation: investigating the molecular correlates of $\mathrm{X}$ chromosome introgression across the hybrid zone between Mus domesticus and Mus musculus. Biol J Linn Soc 2005, 84(3):523-534

22. Piálek J, Barton NH: The spread of an advantageous allele across a barrier: the effects of random drift and selection against heterozygotes. Genetics 1997, 145(2):493-504. 
23. Kim M, Cui ML, Cubas P, Gillies A, Lee K, Chapman MA, Abbott RJ, Coen E: Regulatory genes control a key morphological and ecological trait transferred between species. Science 2008, 322(5904):1116-1119.

24. Whitney KD, Randell RA, Rieseberg LH: Adaptive introgression of abiotic tolerance traits in the sunflower Helianthus annuus. New Phytol 2010, 187(1):230-239.

25. Fitzpatrick BM, Johnson JR, Kump DK, Shaffer HB, Smith JJ, Voss SR: Rapid fixation of non-native alleles revealed by genome-wide SNP analysis of hybrid Tiger Salamanders. BMC Evol Biol 2009, 9:176.

26. Song Y, Endeplos S, Klemann N, Richter D, Matuschka F, Shih C-H, Nachman $\mathrm{MW}$, Kohn MH: Adaptive introgression of anticoagulant rodent poison resistance by hybridization between old world mice. Curr Biol 2011, 21(15):1296-1301.

27. Pardo-Dieaz C, Salazar C, Baxter SW, Merot C, Figueiredo-Ready W, Joron M, McMillan WO, Jiggins CD: Adaptive Introgression across Species Boundaries in Heliconius Butterflies. PLoS Genet 2012, 8(6):e1002752.

28. Putnam AS, Scriber JM, Andolfatto P: Discordant divergence times among Z-chromosome regions between two ecologically distinct swallowtail butterfly species. Evolution 2007, 61(4):912-927.

29. Mao XG, Zhu GJ, Zhang SY, Rossiter SJ: Pleistocene climatic cycling drives intra-specific diversification in the intermediate horseshoe bat (Rhinolophus affinis) in Southern China. Mol Ecol 2010, 19(13):2754-2769.

30. Mao XG, He GM, Hua PY, Jones G, Zhang SY, Rossiter SJ: Historical introgression and the persistence of ghost alleles in the intermediate horseshoe bat (Rhinolophus affinis). Mol Ecol 2013, 22(4):1035-1050.

31. Jones $\mathrm{G}$, Teeling EC, Rossiter SJ: From the ultrasonic to the infrared: molecular evolution and the sensory biology of bats. Front Physiol 2013, 4:117.

32. Li G, Wang JH, Rossiter SJ, Jones G, Zhang SY: Accelerated FoxP2 evolution in echolocating bats. PLOS ONE 2007, 2(9):e900.

33. Li G, Wang JH, Rossiter SJ, Jones G, Cotton JA, Zhang SY: The hearing gene Prestin reunites echolocating bats. P Natl Acad Sci USA 2008, 105(37):13909-13964.

34. Liu Y, Han NJ, Franchini LF, Xu H, Pisciottano F, Elgoyhen AB, Rajan KE, Zhang SY: The voltage-gated potassium channel subfamily KQT member 4 (KCNQ4) displays parallel evolution in echolocating bats. Mol Biol Evol 2012, 29(5):1441-1450.

35. Coyne JA, Orr HA: Speciation. Sunderland, Massachusetts: Sinauer Associates; 2004.

36. Kingston T, Lara MC, Jones G, Akbar Z, Kunz TH, Schneider CJ: Acoustic divergence in two cryptic Hipposideros species: a role for social selection? Proc R Soc London B Biol Sci 2001, 268(1474):1381-1386.

37. Mao XG, Zhang JP, Zhang SY, Rossiter SJ: Historical male-mediated introgression in horseshoe bats revealed by multi-locus DNA sequence data. Mol Ecol 2010, 19(7):1352-1366.

38. Matthee CA, BurzlaV JD, Taylor JF, Davis SK: Mining the mammalian genome for artiodactyl systematics. Syst Biol 2001, 50(3):367-390.

39. Eick GN, Jacobs DS, Matthee CA: A nuclear DNA phylogenetic perspective on the evolution of echolocation and historical biogeography of extant bats (Chiroptera). Mol Biol Evol 2005, 22(9):1869-1886.

40. Lim BK, Engstrom MD, Bickham JW, Patton JC: Molecular phylogeny of New World sheath-tailed bats (Emballonuridae: Diclidurini) based on loci from the four genetic transmission systems in mammals. Biol J Linn SoC 2008, 93(1):189-209.

41. Stephens $M$, Smith N, Donnelly P: A new statistical method for haplotype reconstruction from population data. Am J Hum Gen 2001 68(4):978-989.

42. Librado P, Rozas J: DnaSP v5: a software for comprehensive analysis of DNA polymorphism data. Bioinformatics 2009, 25(11):1451-1452.

43. Thompson JD, Gibson TJ, Plewniak F, Jeanmougin F, Higgins DG: The CLUSTAL_X windows interface: flexible strategies for multiple sequence alignment aided by quality analysis tools. Nucleic Acids Res 1997, 25(24):4876-4882.

44. Ronquist F, Huelsenbeck JP: MrBayes 3: Bayesian phylogenetic inference under mixed models. Bioinformatics 2003, 19(12):1572-1574.

45. Posada D, Crandall KA: MODELTEST: testing the model of DNA substitution. Bioinformatics 1998, 14(9):817-818.

46. Posada D, Crandall KA: Intraspecific gene genealogies: trees grafting into networks. Trends Ecol Evol 2001, 16(1):37-45.
47. Clement M, Posada D, Crandall KA: TCS: a computer program to estimate gene genealogies. Mol Ecol 2000, 9(10):1657-1660.

48. Rosenberg NA: The probability of topological concordance of gene trees and species trees. Theor Popul Biol 2002, 61(2):225-247.

49. Liu L: BEST: Bayesian estimation of species trees under the coalescent model. Bioinformatics 2008, 24(21):2542-2543.

50. Liu L, Edwards SV: Phylogenetic analysis in the anomaly zone. Syst Biol 2009, 58(4):452-460

51. Weir BS, Cockerham CC: Estimating F-statistics for the analysis of population structure. Evolution 1984, 38(6):1358-1370.

52. Excoffier $L$, Lischer HEL: Arlequin suite ver 3.5: a new series of programs to perform population genetics under Linux and Windows. Mol Ecol Resour 2010, 10(3):564-567.

53. Tajima F: Statistical method for testing the neutral mutation hypothesis by DNA polymorphism. Genetics 1989, 123(3):585-595.

54. Hudson RR, Kreitman M, Aguade M: A test of neutral molecular evolution based on nucleotide data. Genetics 1987, 116(1):153-159.

55. Knowles LL, Maddison WP: Statistical phylogeography. Mol Ecol 2002, 11(12):2623-2635.

56. Galbreath KE, Hafner DJ, Zamudio KR, Agnew K: Isolation and introgression in the intermountain West: contrasting gene genealogies reveal the complex biogeographic history of the American pika (Ochotona princeps). J Biogeogr 2010, 37(2):344-362.

57. Maddison WP, Maddison DR: Mesquite: a modular system for evolutionary analysis, v2.5. Available at: http://mesquiteproject.org; 2008.

58. Slatkin M, Maddison WP: A cladistic measure of gene flow inferred from the phylogenies of alleles. Genetics 1989, 123(3):603-613.

59. Swofford DL: PAUP*. Phylogenetic Analysis Using Parsimony ( ${ }^{*}$ and Other Methods). Version 4.0.B10. Sunderland, Massachustts: Sinauer \& Associates; 2002.

60. Beerli P: Comparison of Bayesian and maximum-likelihood inference of population genetic parameters. Bioinformatics 2006, 22(3):341-345.

61. Nielsen $R$, Wakeley J: Distinguishing migration from isolation: a Markov-chain Monte Carlo approach. Genetics 2001, 158(2):885-896.

62. Hey J, Nielsen R: Integration within the Felsenstein equation for improved Markov chain Monte Carlo methods in population genetics. P Natl Acad Sci USA 2007, 104(8):2785-2790.

63. Hey J: The divergence of chimpanzee species and subspecies as revealed in multi-population isolation-with-migration analyses. $\mathrm{Mol} \mathrm{Biol}$ Evol 2010, 27(4):921-933.

64. Hudson RR, Kaplan N: Statistical properties of the number of recombination events in the history of a sample of DNA sequences. Genetics 1985, 111(1):147-164.

65. Takahashi A, Liu YH, Saitou N: Genetic variation versus recombination rate in a structured population of mice. Mol Biol Evol 2004, 21(2):404-409.

66. Keinan A, Reich D: Human population differentiation is strongly correlated with local recombination rate. PLOS Genet 2010, 6(3):e100088655.

67. Carneiro M, Blanco-Aguiar J, Villafuerte R, Ferrand N, Nachman MW: Speciation in the European rabbit (Oryctolagus cuniculus): Islands of differentiation on the $\mathrm{X}$ chromosome and autosomes. Evolution 2010, 64(12):3443-3460.

68. Geraldes A, Basset $P$, smith $K L$, Nachman MW: Higher differentiation among subspecies of the house mouse (Mus musculus) in genomic regions with low recombination. Mol Ecol 2011, 20(22):4722-4736.

69. Herrig DK, Modrick AJ, Brud E, Llopart A: Introgression in the Drosophila subobscura- $D$. Madeirensis sister species: evidence of gene flow in nuclear genes despite mitochondrial differentiation. Evolution 2014, 68(3):705-719

70. Borge T, Webster MT, Andersson G, Sætre GP: Contrasting patterns of polymorphism and divergence on the $\mathrm{Z}$ chromosome and autosomes in two Ficedula flycatcher species. Genetics 2005, 171(4):1861-1873.

71. Macholán $M$, Munclinger $P$, Šugerková $M$, Dufková $P$, Bímová $B$, Božíková E, Zima J, Piálek J: Genetic analysis of autosomal and X-linked markers across a mouse hybrid zone. Evolution 2007, 61(4):746-771.

72. Arnold ML, Martin NH: Adaptation by introgression. J Bio/ 2009, 8:82

73. Hedrick PW: Adaptive introgression in animals: examples and comparison to new mutation and standing variation as sources of adaptive variation. Mol ECOl 2013, 22(18):4606-4618. 
74. Hamilton JA, Lexer C, Aitken SN: Differential introgression reveals candidate genes for selection across a spruce (Picea sitchensis $\mathrm{x}$ P. glauca) hybrid zone. New Phytol 2013, 197(3):927-938.

75. Larson E, Andrés JA, Bogdanowicz SM, Harrison RG: Differential introgression in a mosaic hybrid zone reveals candidate barrier genes. Evolution 2013, 67(12):3653-3661.

76. Roux C, Fraïsse C, Castric V, Vekemans X, Pogson GH, Bierne N: Can we continue to neglect genomic variation in introgression rates when inferring the history of speciation? A case study in Mytilus hybrid zone. $J$ Evol Biol 2014. in press.

doi:10.1186/1471-2148-14-154

Cite this article as: Mao et al:: Differential introgression among loci across a hybrid zone of the intermediate horseshoe bat (Rhinolophus affinis). BMC Evolutionary Biology 2014 14:154.

\section{Submit your next manuscript to BioMed Central and take full advantage of:}

- Convenient online submission

- Thorough peer review

- No space constraints or color figure charges

- Immediate publication on acceptance

- Inclusion in PubMed, CAS, Scopus and Google Scholar

- Research which is freely available for redistribution 